\title{
Some Fixed Point Theorems for Multivalued Maps Satisfying an Implicit Relation on Metrically Convex Spaces
}

\author{
ISHAK ALTUN \\ Department of Mathematics, Faculty of Science and Arts, Kirikkale University, \\ 71450 Yahsihan, Kirikkale, Turkey \\ e-mail : ialtun@kku.edu.tr, ishakaltun@yahoo.com \\ DURAN TURKOGLU \\ Department of Mathematics, Faculty of Science and Arts, Gazi University, 06500 \\ Teknikokullar, Ankara Turkey \\ e-mail : dturkoglu@gazi.edu.tr
}

ABstract. In this paper, we give some fixed point theorems for multivalued maps satisfying an implicit relation on metrically convex spaces. Our results extend and generalize some fixed point theorem in the literature.

\section{Introduction}

In recent years several fixed point results have been obtained on metrically convex spaces. Assad and Kirk [6] gave a sufficient condition enunciating fixed point of set-valued mappings enjoying specific boundary condition in metrically convex metric spaces. A significant generalization of the fixed point theorem of Assad [5] and the theorem of Assad and Kirk [6] for multivalued contraction non-self mappings is obtained by Itoh [17] in 1977. In the current years the work due to Assad and Kirk [6] has inspired extensive activities which includes Ahmad and Imdad [1], [2], Imdad et al. [13], Imdad and Ali [14], Itoh [17], Khan [19] and some others. Most recently, Dhage et al. [9] and Huang and Cho [12] proved some fixed point theorems for a sequence of set-valued mappings which generalize several results due to Ahmad and Khan [3], Itoh [17], Khan [19] and others. The purpose of this paper is to prove some coincidence and common fixed point theorems for multivalued mappings satisfying an implicit relation on metrically convex spaces. Our results either partially or completely generalize earlier results due to Ahmad and Imdad [1], [2], Ahmad and Khan [3], Ćirić [7], Imdad and Khan [15], Itoh [17], Khan [19], Khan et al. [20], Rhoades [26] and several others. See also the related Theorem 3.1 of [14].

Received December 7, 2006.

2000 Mathematics Subject Classification: 54H25, $47 \mathrm{H} 10$.

Key words and phrases: fixed point, multivalued maps, implicit relation, metrically convex space. 


\section{Preliminaries}

Let $(X, d)$ be a metric space. Then $X$ said to be metrically convex if for every pair $x, y \in X, x \neq y$, there is a point $z \in X$ such that $d(x, y)=d(x, z)+d(z, y)$.

Lemma $1([6])$. Let $K$ be non-empty and closed subset of a metrically convex metric space $X$. Then for any $x \in K$ and $y \notin K$, there exists a point $z \in \delta K$ such that $d(x, y)=d(x, z)+d(z, y)$, where $\delta K$ denotes the boundary of $K$.

Let $C B(X)$ denotes the family of all non-empty closed and bounded subsets of $X$. Denote for $A, B \in C B(X)$

$$
d(x, A)=\inf \{d(x, a): a \in A\}
$$

and

$$
H(A, B)=\max \left\{\sup _{x \in A} d(x, B), \sup _{y \in B} d(y, A)\right\} .
$$

The function $H$ is a metric on $C B(X)$ and is called Hausdorff metric. It is well known that if $X$ is a complete metric space, then so is the metric space $(C B(X), H)$.

Lemma 2([21]). Let $A, B \in C B(X)$ and $a \in A$, then for any positive number $q<1$ there exists $b=b(a)$ in $B$ such that $q d(a, b) \leq H(A, B)$.

Definition 1([15]). Let $K$ be a nonempty subset of a metric space $(X, d), T$ : $K \rightarrow X$ and $F: K \rightarrow C B(X)$. The pair $(F, T)$ is said to be pointwise $R$-weakly commuting on $K$ if for given $x \in K$ and $T x \in K$, there exists some $R=R(x)>0$ such that $d(T y, F T x) \leq R d(T x, F x)$ for each $y \in K \cap F x$.

Moreover, the pair $(F, T)$ will be called $R$-weakly commuting on $K$ if $d(T y, F T x) \leq$ $R d(T x, F x)$ holds for each $x \in K, T x \in K$ with some $R>0$.

If $R=1$, we get the definition of weak commutativity of $(F, T)$ on $K$ due to Hadzic and Gajic [11]. For $K=X$ Definition 1 reduces to "pointwise $R$-weak commutativity and $R$-weak commutativity" for single valued self mappings due to Pant [22].

Definition 2([10],[11]). Let $K$ be a nonempty subset of a metric space $(X, d), T$ : $K \rightarrow X$ and $F: K \rightarrow C B(X)$. The pair $(F, T)$ is said to be weakly commuting if for every $x, y \in K$ with $x \in F y$ and $T y \in K$, we have $d(T x, F T y) \leq d(T y, F y)$, whereas the pair $(F, T)$ is said to be compatible if for every sequence $\left\{x_{n}\right\} \subset K$, from the relation $\lim _{n \rightarrow \infty} d\left(F x_{n}, T x_{n}\right)=0$ and $T x_{n} \in K$ (for every $n \in N$ ) it follows that $\lim _{n \rightarrow \infty} d\left(T y_{n}, F T x_{n}\right)=0$ for every sequence $\left\{y_{n}\right\} \subset K$ such that $y_{n} \in F x_{n}, n \in N$.

For hybrid pairs of self type mappings these definitions were introduced by Kaneko and Sessa [18].

Definition 3([13]). Let $K$ be a nonempty subset of a metric space $(X, d), T$ : $K \rightarrow X$ and $F: K \rightarrow C B(X)$. The pair $(F, T)$ is said to be quasi-coincidentally 
commuting if for all coincidence points " $x$ " of $(T, F), T F x \subset F T x$ whenever $F x \subset$ $K$ and $T x \in K$ for all $x \in K$.

Definition 4([13]). A mapping $T: K \rightarrow X$ is said to be coincidentally idempotent w.r.t mapping $F: K \rightarrow C B(X)$, if $T$ is idempotent at the coincidence points of the pair $(F, T)$.

\section{Implicit relation}

Implicit relations on metric space have been used in many articles (see [4], [16], [23], [24], [25], [27]).

Let $R_{+}$denote the set of all non-negative real numbers and let $\mathcal{G}$ be the set of all continuous functions $G: R_{+}^{5} \rightarrow R$ satisfying the following conditions:

$\left(G_{1}\right): G\left(t_{1}, \cdots, t_{5}\right)$ is non-decreasing in $t_{1}$ and non-increasing in $t_{2}, \cdots, t_{5}$.

$\left(G_{2}\right)$ : there exist three constants $a, b \geq 0,2 a+3 b<q<1$ such that the inequality

$$
G(u, v, w, v, v+w) \leq 0
$$

implies $u \leq \max \{(a+b) v+b w,(a+b) w+b v\}$.

$\left(G_{3}\right): G(q u, u, 0,0,2 u)>0, \forall u>0$.

Now we give some examples. In the following examples, the condition $\left(G_{1}\right)$ is obvious.

Example 1. Let $G\left(t_{1}, \cdots, t_{5}\right)=t_{1}-\alpha \max \left\{\frac{t_{2}}{2}, t_{3}, t_{4}\right\}-\beta t_{5}$, where $\alpha, \beta \geq 0, \alpha>2 \beta$ and $2 \alpha+3 \beta<q<1$.

Let $G(u, v, w, v, v+w)=u-\alpha \max \{w, v\}-\beta(w+v) \leq 0$. Thus $u \leq \max \{(\alpha+$ $\beta) v+\beta w,(\alpha+\beta) w+\beta v\}$ and so $\left(G_{2}\right)$ is satisfied with $a=\alpha$ and $b=\beta$.

$G(q u, u, 0,0,2 u)=u\left(q-\frac{\alpha}{2}-2 \beta\right)>0, \forall u>0$.

Therefore $G \in \mathcal{G}$.

Example 2. Let $G\left(t_{1}, \cdots, t_{5}\right)=t_{1}-\alpha t_{2}-\beta\left(t_{3}+t_{4}\right)-\gamma t_{5}$, where $\alpha, \beta, \gamma \geq 0$ and $2 \alpha+3 \beta+3 \gamma<q<1$.

Let $G(u, v, w, v, v+w)=u-\alpha v-\beta(w+v)-\gamma(w+v) \leq 0$. Thus $u \leq(\alpha+\beta+$ $\gamma) v+(\beta+\gamma) w \leq \max \{(\alpha+\beta+\gamma) v+(\beta+\gamma) w,(\alpha+\beta+\gamma) w+(\beta+\gamma) v\}$ and so $\left(G_{2}\right)$ is satisfied with $a=\alpha, b=\beta+\gamma$.

$G(q u, u, 0,0,2 u)=u(q-\alpha-2 \gamma), \forall u>0$.

Therefore $G \in \mathcal{G}$.

Example 3. Let $G\left(t_{1}, \cdots, t_{5}\right)=t_{1}-\alpha t_{2}-\beta \max \left\{t_{3}+t_{4}, t_{5}\right\}$, where $\alpha, \beta, \gamma \geq 0$ and $2 \alpha+3 \beta<q<1$.

Let $G(u, v, w, v, v+w)=u-\alpha v-\beta(v+w) \leq 0$. Thus $u \leq(\alpha+\beta) v+\beta w \leq$ $\max \{(\alpha+\beta) v+\beta w,(\alpha+\beta) w+\beta v\}$ and so $\left(G_{2}\right)$ is satisfied with $a=\alpha, b=\beta$.

$G(q u, u, 0,0,2 u)=u(q-\alpha-2 \beta), \forall u>0$.

Therefore $G \in \mathcal{G}$. 
Example 4. Let $G\left(t_{1}, \cdots, t_{5}\right)=t_{1}-\alpha t_{2}-\beta \max \left\{t_{3}, t_{4}\right\}-\gamma t_{5}$, where $\alpha, \beta, \gamma \geq 0$ and $2 \alpha+3 \beta+3 \gamma<q<1$.

Let $G(u, v, w, v, v+w)=u-\alpha v-\beta \max \{w, v\}-\gamma(w+v) \leq 0$. Thus $u \leq$ $\max \{(\alpha+\beta+\gamma) v+(\beta+\gamma) w,(\alpha+\beta+\gamma) w+(\beta+\gamma) v\}$ and so $\left(G_{2}\right)$ is satisfied with $a=\alpha, b=\beta+\gamma$

$G(q u, u, 0,0,2 u)=u(q-\alpha-2 \gamma)>0, \forall u>0$.

Therefore $G \in \mathcal{G}$.

Example 5. Let $G\left(t_{1}, \cdots, t_{5}\right)=t_{1}-\max \left\{\frac{\alpha t_{3} t_{5}}{t_{3}+t_{5}+1}, \frac{\beta t_{4} t_{5}}{t_{4}+t_{5}+1}\right\}-\gamma\left(t_{3}+t_{4}\right)$, where $\alpha, \beta, \gamma \geq 0,2 \alpha+2 \beta+3 \gamma<q<1$.

Let $G(u, v, w, v, v+w)=u-\max \left\{\frac{\alpha v(v+w)}{v+2 w+1}, \frac{\beta w(v+w)}{2 v+w+1}\right\}-\gamma(v+w) \leq 0$. Thus

$$
\begin{aligned}
u & \leq \max \left\{\frac{\alpha v(v+w)}{v+2 w+1}, \frac{\beta w(v+w)}{2 v+w+1}\right\}+\gamma(v+w) \\
& \leq \max \{\alpha v, \beta w\}+\gamma(v+w) \\
& =\max \{(\alpha+\gamma) v+\gamma w,(\beta+\gamma) w+\gamma v\} \\
& \leq \max \{(\alpha+\beta+\gamma) v+\gamma w,(\alpha+\beta+\gamma) w+\gamma v\}
\end{aligned}
$$

and so $\left(G_{2}\right)$ is satisfied with $a=\alpha+\beta, b=\gamma$.

$G(q u, u, 0,0,2 u)=q u>0, \forall u>0$.

Therefore $G \in \mathcal{G}$.

\section{Main result}

Now we give our main theorem.

Theorem 1. Let $(X, d)$ be a metrically convex complete metric space and $K$ a non-empty closed subset of $X$. Let $\left\{F_{n}\right\}_{n=1}^{\infty}: K \rightarrow C B(X)$ and $S, T: K \rightarrow X$ satisfying

(a) $\delta K \subseteq S K \cap T K, F_{i}(K) \cap K \subseteq S K, F_{j}(K) \cap K \subseteq T K$,

(b) $T x \in \delta K \Rightarrow F_{i}(x) \subseteq K, S x \in \delta K \Rightarrow F_{j}(x) \subseteq K$, and

$$
\begin{array}{r}
G\left(H\left(F_{i}(x), F_{j}(y)\right), d(T x, S y), d\left(T x, F_{i}(x)\right), d\left(S y, F_{j}(y)\right),\right. \\
\left.d\left(T x, F_{j}(y)\right)+d\left(S y, F_{i}(x)\right)\right) \leq 0
\end{array}
$$

for all $x, y \in K$, where $G \in \mathcal{G}, i=2 n-1, j=2 n,(n \in N)$,

(c) $\left(F_{i}, T\right)$ and $\left(F_{j}, S\right)$ are compatible pairs,

(d) $\left\{F_{n}\right\}, S$ and $T$ are continuous on $K$.

Then $\left(F_{i}, T\right)$ as well as $\left(F_{j}, S\right)$ has a point of coincidence.

Proof. Firstly, we proceed to construct two sequences $\left\{x_{n}\right\}$ and $\left\{y_{n}\right\}$ in the following way.

Let $x \in \delta K$. Then (due to $\delta K \subseteq T K$ ) there exists a point $x_{0} \in K$ such that $x=T x_{0}$. From the implication $T x \in \delta K$ which implies $F_{1}\left(x_{0}\right) \subseteq F_{1}(K) \cap K \subseteq S K$, 
let $x_{1} \in K$ be such that $y_{1}=S x_{1} \in F_{1}\left(x_{0}\right) \subseteq K$. Since $y_{1} \in F_{1}\left(x_{0}\right)$, there exists a point $y_{2} \in F_{2}\left(x_{1}\right)$ such that

$$
q d\left(y_{1}, y_{2}\right) \leq H\left(F_{1}\left(x_{0}\right), F_{2}\left(x_{1}\right)\right) .
$$

Suppose $y_{2} \in K$. Then $y_{2} \in F_{2}(K) \cap K \subseteq T K$ implies that there exists a point $x_{2} \in K$ such that $y_{2}=T x_{2}$. Otherwise, if $y_{2} \notin K$, then there exists a point $p \in \delta K$ such that

$$
d\left(S x_{1}, p\right)+d\left(p, y_{2}\right)=d\left(S x_{1}, y_{2}\right) .
$$

Since $p \in \delta K \subseteq T K$, there exists a point $x_{2} \in K$ with $p=T x_{2}$ so that

$$
d\left(S x_{1}, T x_{2}\right)+d\left(T x_{2}, y_{2}\right)=d\left(S x_{1}, y_{2}\right) .
$$

$y_{3} \in F_{3}\left(x_{2}\right)$ be such that $q d\left(y_{2}, y_{3}\right) \leq H\left(F_{2}\left(x_{1}\right), F_{3}\left(x_{2}\right)\right)$.

Thus, repeating the foregoing arguments, we obtain two sequences $\left\{x_{n}\right\}$ and $\left\{y_{n}\right\}$ such that

(e) $y_{2 n} \in F_{2 n}\left(x_{2 n-1}\right), y_{2 n+1} \in F_{2 n+1}\left(x_{2 n}\right)$,

(f) $y_{2 n} \in K \Rightarrow y_{2 n}=T x_{2 n}$ or $y_{2 n} \notin K \Rightarrow T x_{2 n} \in \delta K$ and

$$
d\left(S x_{2 n-1}, T x_{2 n}\right)+d\left(T x_{2 n}, y_{2 n}\right)=d\left(S x_{2 n-1}, y_{2 n}\right),
$$

(g) $y_{2 n+1} \in K \Rightarrow y_{2 n+1}=S x_{2 n+1}$ or $y_{2 n+1} \notin K \Rightarrow S x_{2 n+1} \in \delta K$ and

$$
d\left(T x_{2 n}, S x_{2 n+1}\right)+d\left(S x_{2 n+1}, y_{2 n+1}\right)=d\left(T x_{2 n}, y_{2 n+1}\right) .
$$

We denote

$$
\begin{aligned}
& P_{0}=\left\{T x_{2 i} \in\left\{T x_{2 n}\right\}: T x_{2 i}=y_{2 i}\right\} \\
& P_{1}=\left\{T x_{2 i} \in\left\{T x_{2 n}\right\}: T x_{2 i} \neq y_{2 i}\right\} \\
& Q_{0}=\left\{S x_{2 i+1} \in\left\{S x_{2 n+1}\right\}: S x_{2 i+1}=y_{2 i+1}\right\} \\
& Q_{1}=\left\{S x_{2 i+1} \in\left\{S x_{2 n+1}\right\}: S x_{2 i+1} \neq y_{2 i+1}\right\} .
\end{aligned}
$$

One can note that $\left(T x_{2 n}, S x_{2 n+1}\right) \notin P_{1} \times Q_{1}$ and $\left(S x_{2 n-1}, T x_{2 n}\right) \notin Q_{1} \times P_{1}$.

Now we distinguish the following three cases.

Case 1. If $\left(T x_{2 n}, S x_{2 n+1}\right) \in P_{0} \times Q_{0}$, then using (4.1), we have

$$
\begin{aligned}
& G\left(H\left(F_{2 n+1}\left(x_{2 n}\right), F_{2 n}\left(x_{2 n-1}\right)\right), d\left(T x_{2 n}, S x_{2 n-1}\right), d\left(T x_{2 n}, F_{2 n+1}\left(x_{2 n}\right)\right),\right. \\
& \left.d\left(S x_{2 n-1}, F_{2 n}\left(x_{2 n-1}\right)\right), d\left(T x_{2 n}, F_{2 n}\left(x_{2 n-1}\right)\right)+d\left(S x_{2 n-1}, F_{2 n+1}\left(x_{2 n}\right)\right)\right) \leq 0
\end{aligned}
$$

or

$$
\begin{array}{r}
G\left(H\left(F_{2 n+1}\left(x_{2 n}\right), F_{2 n}\left(x_{2 n-1}\right)\right), d\left(y_{2 n}, y_{2 n-1}\right), d\left(y_{2 n}, y_{2 n+1}\right), d\left(y_{2 n-1}, y_{2 n}\right),\right. \\
\left.d\left(y_{2 n-1}, y_{2 n}\right)+d\left(y_{2 n}, y_{2 n+1}\right)\right) \leq 0 .
\end{array}
$$

From $\left(G_{2}\right)$, there exist three constants $a, b \geq 0,2 a+3 b<q<1$ such that

$$
\begin{aligned}
& H\left(F_{2 n+1}\left(x_{2 n}\right), F_{2 n}\left(x_{2 n-1}\right)\right) \\
\leq & \max \left\{\begin{array}{c}
(a+b) d\left(y_{2 n-1}, y_{2 n}\right)+b d\left(y_{2 n}, y_{2 n+1}\right), \\
(a+b) d\left(y_{2 n}, y_{2 n+1}\right)+b d\left(y_{2 n-1}, y_{2 n}\right)
\end{array} .\right.
\end{aligned}
$$


Since

$$
\begin{aligned}
q d\left(y_{2 n}, y_{2 n+1}\right) & =q d\left(T x_{2 n}, S x_{2 n+1}\right) \\
& \leq H\left(F_{2 n+1}\left(x_{2 n}\right), F_{2 n}\left(x_{2 n-1}\right)\right),
\end{aligned}
$$

from (4.2), we have

$$
d\left(y_{2 n}, y_{2 n+1}\right) \leq \max \left\{\frac{a+b}{q-b}, \frac{b}{q-a-b}\right\} d\left(y_{2 n-1}, y_{2 n}\right)
$$

or

$$
d\left(T x_{2 n}, S x_{2 n+1}\right) \leq \max \left\{\frac{a+b}{q-b}, \frac{b}{q-a-b}\right\} d\left(S x_{2 n-1}, T x_{2 n}\right) .
$$

Note that $\max \left\{\frac{a+b}{q-b}, \frac{b}{q-a-b}\right\}<1$ since $2 a+3 b<q<1$.

Similarly if $\left(S x_{2 n-1}, T x_{2 n}\right) \in P_{0} \times Q_{1}$, then

$$
d\left(S x_{2 n-1}, T x_{2 n}\right) \leq \max \left\{\frac{a+b}{q-b}, \frac{b}{q-a-b}\right\} d\left(S x_{2 n-1}, T x_{2 n-2}\right) .
$$

Case 2. If $\left(T x_{2 n}, S x_{2 n+1}\right) \in P_{0} \times Q_{1}$, then

$$
d\left(T x_{2 n}, S x_{2 n+1}\right)+d\left(S x_{2 n+1}, y_{2 n+1}\right)=d\left(T x_{2 n}, y_{2 n+1}\right)
$$

which in turn yields

$$
d\left(T x_{2 n}, S x_{2 n+1}\right) \leq d\left(T x_{2 n}, y_{2 n+1}\right)=d\left(y_{2 n}, y_{2 n+1}\right)
$$

and hence

$$
q d\left(T x_{2 n}, S x_{2 n+1}\right) \leq q d\left(y_{2 n}, y_{2 n+1}\right) \leq H\left(F_{2 n+1}\left(x_{2 n}\right), F_{2 n}\left(x_{2 n-1}\right)\right) .
$$

Now, proceeding as in Case 1, we have

$$
d\left(T x_{2 n}, S x_{2 n+1}\right) \leq \max \left\{\frac{a+b}{q-b}, \frac{b}{q-a-b}\right\} d\left(S x_{2 n-1}, T x_{2 n}\right) .
$$

In case $\left(S x_{2 n-1}, T x_{2 n}\right) \in Q_{1} \times P_{0}$, then as earlier, one also obtains

$$
d\left(S x_{2 n-1}, T x_{2 n}\right) \leq \max \left\{\frac{a+b}{q-b}, \frac{b}{q-a-b}\right\} d\left(S x_{2 n-1}, T x_{2 n-2}\right) .
$$

Case 3. If $\left(T x_{2 n}, S x_{2 n+1}\right) \in P_{1} \times Q_{0}$, then $S x_{2 n-1}=y_{2 n-1}$ and

$$
\begin{aligned}
q d\left(T x_{2 n}, S x_{2 n+1}\right) & =q d\left(T x_{2 n}, y_{2 n+1}\right) \\
& \leq q d\left(T x_{2 n}, y_{2 n}\right)+q d\left(y_{2 n}, y_{2 n+1}\right) \\
& \leq q d\left(T x_{2 n}, y_{2 n}\right)+H\left(F_{2 n+1}\left(x_{2 n}\right), F_{2 n}\left(x_{2 n-1}\right)\right) .
\end{aligned}
$$


Now again using (4.1), we have

$$
\begin{aligned}
& G\left(H\left(F_{2 n+1}\left(x_{2 n}\right), F_{2 n}\left(x_{2 n-1}\right)\right), d\left(T x_{2 n}, S x_{2 n-1}\right), d\left(T x_{2 n}, F_{2 n+1}\left(x_{2 n}\right)\right),\right. \\
& \left.d\left(S x_{2 n-1}, F_{2 n}\left(x_{2 n-1}\right)\right), d\left(T x_{2 n}, F_{2 n}\left(x_{2 n-1}\right)\right)+d\left(S x_{2 n-1}, F_{2 n+1}\left(x_{2 n}\right)\right)\right) \leq 0
\end{aligned}
$$

or

$$
\begin{array}{r}
G\left(H\left(F_{2 n+1}\left(x_{2 n}\right), F_{2 n}\left(x_{2 n-1}\right)\right), d\left(y_{2 n}, y_{2 n-1}\right), d\left(y_{2 n}, y_{2 n+1}\right)\right. \\
\left.d\left(y_{2 n-1}, y_{2 n}\right), d\left(y_{2 n-1}, y_{2 n}\right)+d\left(y_{2 n}, y_{2 n+1}\right)\right) \leq 0
\end{array}
$$

From $\left(G_{2}\right)$,

$$
\begin{aligned}
& H\left(F_{2 n+1}\left(x_{2 n}\right), F_{2 n}\left(x_{2 n-1}\right)\right) \\
& \leq \max \left\{\begin{array}{c}
(a+b) d\left(y_{2 n-1}, y_{2 n}\right)+b d\left(y_{2 n}, y_{2 n+1}\right) \\
(a+b) d\left(y_{2 n}, y_{2 n+1}\right)+b d\left(y_{2 n-1}, y_{2 n}\right)
\end{array}\right.
\end{aligned}
$$

From (4.3) and (4.4), we have

$$
d\left(T x_{2 n}, S x_{2 n+1}\right) \leq \max \left\{\frac{q+b}{q-a-b}, \frac{q+a+b}{q-b}\right\} d\left(T x_{2 n}, S x_{2 n-1}\right) .
$$

Now, proceeding as earlier, one also obtains

$$
d\left(S x_{2 n-1}, T x_{2 n}\right) \leq \max \left\{\frac{a+b}{q-b}, \frac{b}{q-a-b}\right\} d\left(S x_{2 n-1}, T x_{2 n-2}\right)
$$

Therefore combining above inequalities, we have

$$
d\left(T x_{2 n}, S x_{2 n+1}\right) \leq k d\left(S x_{2 n-1}, T x_{2 n-2}\right),
$$

where

$$
k=\max \left\{\begin{array}{c}
\left(\frac{a+b}{q-b}\right)\left(\frac{q+b}{q-a-b}\right),\left(\frac{a+b}{q-b}\right)\left(\frac{q+a+b}{q-b}\right), \\
\left(\frac{b}{q-a-b}\right)\left(\frac{q+b}{q-a-b}\right),\left(\frac{b}{q-a-b}\right)\left(\frac{q+a+b}{q-b}\right)
\end{array}\right\}<1
$$

since $2 a+3 b<q<1$.

To see (4.5), $2 a+3 b<q<1$ yields

$$
\begin{aligned}
& a+b<q-a-2 b \\
\Rightarrow & a q+b q<q^{2}-a q-2 b q \\
\Rightarrow & a q+b q+a b+b^{2}<q^{2}-a q-2 b q+a b+b^{2} \\
\Rightarrow & (a+b)(q+b)<(q-b)(q-a-b) \\
\Rightarrow & \frac{(a+b)(q+b)}{(q-b)(q-a-b)}<1
\end{aligned}
$$


and

$$
\begin{aligned}
& 3 b<q \\
\Rightarrow & \frac{q}{q-b}<\frac{3}{2} \\
\Rightarrow & \frac{q}{2(q-b)}+\frac{1}{4}<1 \\
\Rightarrow & \frac{1}{2}\left(\frac{q}{(q-b)}+\frac{1}{2}\right)<1 \\
\Rightarrow & \frac{a+b}{q-b}\left(\frac{q}{q-b}+\frac{a+b}{q-b}\right)<1 \\
\Rightarrow & \frac{(a+b)(q+a+b)}{(q-b)^{2}}<1
\end{aligned}
$$

and

$$
\begin{aligned}
& b<q-2 a-2 b \\
\Rightarrow & b q<q^{2}-2 a q-2 b q \\
\Rightarrow & b q+b^{2}<q^{2}-2 a q-2 b q+b^{2}+a^{2}+2 a b \\
\Rightarrow & b(q+b)<(q-a-b)^{2} \\
\Rightarrow & \frac{b(q+b)}{(q-a-b)^{2}}<1
\end{aligned}
$$

and

$$
\begin{aligned}
& a+3 b<q \\
\Rightarrow & a q+3 b q<q^{2} \\
\Rightarrow & b q<q^{2}-a q-2 b q \\
\Rightarrow & b q+a b+b^{2}<q^{2}-a q-2 b q+a b+b^{2} \\
\Rightarrow & b(q+a+b)<(q-a-b)(q-b) \\
\Rightarrow & \frac{b(q+a+b)}{(q-a-b)(q-b)}<1 .
\end{aligned}
$$

Thus in all the cases, we have

$$
d\left(T x_{2 n}, S x_{2 n+1}\right) \leq k \max \left\{d\left(S x_{2 n-1}, T x_{2 n}\right), d\left(T x_{2 n-2}, S x_{2 n-1}\right)\right\}
$$

whereas

$$
d\left(S x_{2 n+1}, T x_{2 n+2}\right) \leq k \max \left\{d\left(S x_{2 n-1}, T x_{2 n}\right), d\left(T x_{2 n}, S x_{2 n+1}\right)\right\} .
$$

Now on the lines of Assad and Kirk [6], it can be shown by induction that for $n \geq 1$, we have

$$
d\left(T x_{2 n}, S x_{2 n+1}\right)<k^{n} \delta, \quad d\left(S x_{2 n+1}, T x_{2 n+2}\right)<k^{n+\frac{1}{2}} \delta
$$

whereas

$$
\delta=k^{-\frac{1}{2}} \max \left\{d\left(T x_{0}, S x_{1}\right), d\left(S x_{1}, T x_{2}\right)\right\} .
$$

Thus the sequence $\left\{T x_{0}, S x_{1}, T x_{2}, S x_{3}, \cdots, S x_{2 n-1}, T x_{2 n}, S x_{2 n+1}, \cdots\right\}$ is Cauchy and hence converges to the point $z$ in $X$. Then as noted in [10] there exists at least one subsequence $\left\{T x_{2 n_{k}}\right\}$ or $\left\{S x_{2 n_{k}+1}\right\}$ which is contained in $P_{0}$ or $Q_{0}$ respectively. 
Suppose that the subsequence $\left\{T x_{2 n_{k}}\right\}$ contained in $P_{0}$ for each $k \in N$ converges to $z$. Using compatibility of $\left(F_{j}, S\right)$, we have

$$
\lim _{k \rightarrow \infty} d\left(S x_{2 n_{k}-1}, F_{j}\left(x_{2 n_{k}-1}\right)\right)=0 \text { for any even integer } j \in N,
$$

which implies that $\lim _{k \rightarrow \infty} d\left(S T x_{2 n_{k}}, F_{j}\left(S x_{2 n_{k}-1}\right)\right)=0$.

Using the continuity of $S$ and $F_{j}$, one obtains $S z \in F_{j}(z)$, for any even integer $j \in N$. Similarly continuity of $T$ and $F_{i}$ implies $T z \in F_{i}(z)$, for any odd integer $i \in N$. Now using (4.1), we have

$$
\begin{array}{r}
G\left(H\left(F_{i}(z), F_{j}(z)\right), d(T z, S z), d\left(T z, F_{i}(z)\right), d\left(S z, F_{j}(z)\right),\right. \\
\left.d\left(T z, F_{j}(z)\right)+d\left(S z, F_{i}(z)\right)\right) \leq 0
\end{array}
$$

or, since $q d(T z, S z) \leq H\left(F_{i}(z), F_{j}(z)\right)$,

$$
G(q d(T z, S z), d(T z, S z), 0,0,2 d(T z, S z)) \leq 0
$$

which is a contradiction with $\left(G_{3}\right)$ if $d(T z, S z)>0$. Thus we obtain $d(T z, S z)=0$ and so $T z=S z$ which shows that $z$ is a common coincidence point of the maps $\left\{F_{n}\right\}, S$ and $T$.

Remark 1. By Theorem 1, we get an improved version of main theorem of [4].

Remark 2. Theorem 3.1 of [15], which is a generalization of results of [1], [2], follows from Example 1 and Theorem 1.

Remark 3. Theorem 1 can prove for pointwise $R$-weakly commuting maps as Theorem 3.4 of [15].

Theorem 2. Let $(X, d)$ be a metrically convex complete metric space and $K$ a nonempty closed subset of $X$. Let $\left\{F_{n}\right\}_{n=1}^{\infty}: K \rightarrow C B(X)$ and $S, T: K \rightarrow X$ satisfying (4.1), (a) and (b). Suppose that

(h) TK and $S K$ are closed subspaces of $X$. Then $\left(F_{i}, T\right)$ has a point of coincidence and $\left(F_{j}, S\right)$ has a point of coincidence.

Moreover, $\left(F_{i}, T\right)$ has a common fixed point if $T$ is quasi-coincidentally commuting and coincidentally idempotent w.r.t. $F_{i}$ whereas $\left(F_{j}, S\right)$ has a common fixed point provided $S$ is quasi-coincidentally commuting and coincidentally idempotent w.r.t. $F_{j}$.

Remark 4. Theorem 3.5 of [15], which is a generalization of results of Khan [19] and Khan et al. [20], follows from Example 1 and Theorem 2.

Theorem 3. Let $(X, d)$ be a metrically convex complete metric space and $K$ a non-empty closed subset of $X$. Let $\left\{F_{n}\right\}_{n=1}^{\infty}: K \rightarrow C B(X)$ satisfying

(i) $x \in \delta K \Rightarrow F_{n}(x) \subseteq K$ and

$$
G\left(H\left(F_{i}(x), F_{j}(y)\right), d(x, y), d\left(x, F_{i}(x)\right), d\left(y, F_{j}(y)\right), d\left(x, F_{j}(y)\right)+d\left(y, F_{i}(x)\right)\right) \leq 0
$$


for all $x, y \in K$, where $G \in \mathcal{G}, i \neq j$,

Then $\left\{F_{n}\right\}$ has a common fixed point.

Remark 5. Corollary 3.6 of [15], which is a generalization of results of [8], follows from Example 1 and Theorem 3.

Remark 6. If we combined Example 5 with Theorem 3, we have the following interesting result.

Corollary 1. Let $(X, d)$ be a metrically convex complete metric space and $K$ a non-empty closed subset of $X$. Let $\left\{F_{n}\right\}_{n=1}^{\infty}: K \rightarrow C B(X)$ satisfying

(j) $x \in \delta K \Rightarrow F_{n}(x) \subseteq K$ and

$$
\begin{aligned}
& H\left(F_{i}(x), F_{j}(y)\right) \leq \max \left\{\begin{array}{c}
\frac{\alpha d\left(x, F_{i}(x)\right)\left[d\left(x, F_{j}(y)\right)+d\left(y, F_{i}(x)\right)\right]}{d\left(x, F_{i}(x)\right)+d\left(x, F_{j}(y)\right)+d\left(y, F_{i}(x)\right)+1}, \\
\frac{\beta d\left(x, F_{i}(x)\right)\left[d\left(x, F_{j}(y)\right)+d\left(y, F_{i}(x)\right)\right]}{d\left(y, F_{j}(y)\right)+d\left(x, F_{j}(y)\right)+d\left(y, F_{i}(x)\right)+1}
\end{array}\right\} \\
&+\gamma\left[d\left(x, F_{i}(x)\right)+d\left(y, F_{j}(y)\right)\right]
\end{aligned}
$$

for all $x, y \in K$, where $\alpha, \beta, \gamma \geq 0,2 \alpha+2 \beta+3 \gamma<q<1, i \neq j$.

Then $\left\{F_{n}\right\}$ has a common fixed point.

Remark 7. We can have some new results, if we combined Theorem 1, Theorem 2 or Theorem 3 with some examples of $G$.

\section{References}

[1] A. Ahmad and M. Imdad, On common fixed point of mappings and multivalued mappings, Rad. Mat., 8(1) (1992), 147-158.

[2] A. Ahmad and M. Imdad, Some common fixed point theorems for mappings and multi-valued mappings, J. Math. Anal. Appl., 218(2) (1998), 546-560.

[3] A. Ahmad and A. R. Khan, Some common fixed point theorems for non-self-hybrid contractions, J. Math. Anal. Appl., 213(1) (1997), 275-286.

[4] I. Altun, H. A. Hancer and D. Turkoglu, A fixed point theorem for multi-maps satisfying an implicit relation on metrically convex metric spaces, Mathematical Commun., 11 (2006), 17-23.

[5] N. A. Assad, Fixed point theorems for set valued transformations on compact sets, Boll. Un. Mat. Ital., 8(4) (1973), 1-7.

[6] N. A. Assad and W. A. Kirk, Fixed point theorems for set-valued mappings of contractive type, Pacific J. Math., 43(3) (1972), 553-562.

[7] Lj. B. Ćirić, Fixed Point Theory, Faculty of Mechanical Engineering University of Belgrade, Beograd, 2003.

[8] Lj. B. Ćirić and J. S. Ume, On an extension of a theorem of Rhoades, Rev. Roumaine Math. Pures Appl., 49(2) (2004), 103-112. 
[9] B. C. Dhage, U. P. Dolhare and A. Petruşel, Some common fixed point theorems for sequences of non-self multivalued operators in metrically convex metric spaces, Fixed Point Theory, 4(2) (2003), 143-158.

[10] O. Hadžić, On coincidence points in convex metric spaces, Univ. u Novom Sadu Zb. Rad. Prirod.-Mat. Fak. Ser. Mat., 19(2) (1989), 233-240.

[11] O. Hadžić and Lj. Gajić, Coincidence points for set-valued mappings in convex metric spaces, Univ. u Novom Sadu Zb. Rad. Prirod.-Mat. Fak. Ser. Mat., 16(1) (1986), 1325 .

[12] N. J. Huang and Y. J. Cho, Common fixed point theorems for a sequence of set-valued mappings, J. Korean Math. Soc., 4(1) (1997), 1-10.

[13] M. Imdad, A. Ahmad, and S. Kumar, On nonlinear nonself hybrid contractions, Rad. Mat., 10(2) (2001), 233-244.

[14] M. Imdad and Javid Ali, A common fixed point theorem for nonself multi-maps satisfying an implicit relation, Global J. Math. Anal., 1 (2007), 74-83.

[15] M. Imdad and L. Khan, Fixed point theorems for a family of hybrid pairs of mappings in metrically convex spaces, Fixed Point Theory Appl., 2005, no. 3, 281-294.

[16] M. Imdad, S. Kumar and M. S. Khan, Remarks on some fixed point theorems satisfying implicit relations, Rad. Math., 11 (1) (2002), 135-143.

[17] S. Itoh, Multivalued generalized contractions and fixed point theorems, Comment. Math. Univ. Carolinae, 18(2) (1977), 247-258.

[18] H. Kaneko and S. Sessa, Fixed point theorems for compatible multi-valued and singlevalued mappings, Int. J. Math. Math. Sci., 12(2) (1989), 257-262.

[19] M. S. Khan, Common fixed point theorems for multivalued mappings, Pacific J. Math., 95(2) (1981), 337-347.

[20] M. S. Khan, H. K. Pathak, and M. D. Khan, Some fixed point theorems in metrically convex spaces, Georgian Math. J., 7(3) (2000), 523-530.

[21] S. B. Nadler Jr., Multi-valued contraction mappings, Pacific J. Math., 30(2) (1969), 475-488.

[22] R. P. Pant, Common fixed points of noncommuting mappings, J. Math. Anal. Appl., 188(2) (1994), 436-440.

[23] V. Popa, Some fixed point theorems for compatible mappings satisfying an implicit relation, Demonsratio Math., 32 (1999), 157-163.

[24] V. Popa, A general coincidence theorem for compatible multivalued mappings satisfying an implicit relation, Demonstratio Math., 33 (2000), 159-164.

[25] V. Popa and D. Turkoglu, Some fixed point theorems for hybrid contractions satisfying an implicit relation, Stud. Cercet. Stiin. Ser. Mat. Univ. Bacau, 1998, no. 8, 75-86 (2000).

[26] Rhoades B. E., A fixed point theorem for a multi-valued non-self mappings, Comment. Math. Univ. Carolinae, 37 (1996), 401-404.

[27] S. Sharma and B. Desphande, On compatible mappings satisfying an implicit relation in common fixed point consideration, Tamkang J. Math., 33(3) (2002), 245-252. 